\title{
A deep dive into NGC 604 with Gemini/NIRI imaging
}

\author{
Cecilia Fariña, ${ }^{1,2}$ Guillermo L. Bosch ${ }^{1,2}$ and Rodolfo R. Barbá ${ }^{3,4}$ \\ ${ }^{1}$ Facultad de Ciencias Astronómicas y Geofísicas, Universidad Nacional de La Plata, \\ Paseo de Bosque s/n, 1900 La Plata, Argentina \\ email: ceciliaf@f caglp.unlp.edu.ar \\ ${ }^{2}$ IALP, CONICET, Argentina \\ ${ }^{3}$ ICATE, CONICET, Argentina \\ ${ }^{4}$ Departamento de Física, Universidad de La Serena, Benavente 980, La Serena, Chile
}

\begin{abstract}
The giant HiI region NGC 604 constitutes a complex and rich population to study in detail many aspects of massive star formation, such as their environments and physical conditions, the evolutionary processes involved, the initial mass function for massive stars and starformation rates, among many others. Here, we present our first results of a near-infrared study of NGC 604 performed with NIRI images obtained with Gemini North. Based on deep JHK photometry, 164 sources showing infrared excess were detected, pointing to the places where we should look for star-formation processes currently taking place. In addition, the color-color diagram reveals a great number of objects that could be giant/supergiant stars or unresolved, small, tight clusters. An extinction map obtained based on narrow-band images is also shown.
\end{abstract}

Keywords. ISM: individual (NGC 604), Hiı regions, galaxies: individual (M 33), techniques: photometric, stars: formation, stars: early-type, infrared: stars

\section{Introduction}

NGC 604 is a giant HII region (GHR) located in an outer spiral arm of M33, at a distance of $840 \mathrm{kpc}$. It is the second most luminous HiI region in the Local Group, after 30 Doradus in the LMC. Both are nearby examples of giant star-forming regions whose individual objects can be spatially resolved for further study. NGC 604 has been the target of many studies during the past few decades. A brief summary of known facts about NGC 604 includes the following. This GHR is ionized by a massive, young cluster, with at least $200 \mathrm{O}$ stars (some as early as O3-O4). The cluster does not exhibit a central core distribution. Instead, the stars are widely spread over its projected area in a structure called 'scaled OB association' (SOBA; Hunter et al. 1996; Maíz Apellániz et al. 2004; Bruhweiler et al. 2003). Wolf-Rayet stars, a confirmed and many candidate red supergiant stars, a luminous blue variable and a supernova remnant are all part of NGC 604's stellar population (Conti \& Massey 1981; D'Odorico \& Rosa 1981; Drissen et al. 1993; Díaz et al. 1996; Churchwell \& Goss 1999; Terlevich et al. 1996; Barbá et al. 2009). The age of the central ionizing cluster has been determined by different authors as between 3 and 5 Myr (González-Delgado \& Pérez 2000; Bruhweiler et al. 2003; Díaz et al. 1996; Hunter et al. 1996; Relaño \& Kennicut 2009).

The interstellar medium reveals a complex structure with a high-excitation central region (made up of multiple two-dimensional structures), asymmetrically surrounded by a low-excitation halo. The whole region shows a very complex geometry of cavities, expanding shells and filaments, as well as dense molecular regions. All of these structures 
Table 1. Main characteristics of broad-band and narrow-band filters used in our Gemini/NIRI observations.

\begin{tabular}{ccc|ccc} 
& \multicolumn{2}{c}{ Broad bands } & \multicolumn{2}{c}{ Narrow bands } \\
Filter & Central $\lambda(\mu \mathrm{m})$ & Coverage $(\mu \mathrm{m})$ & Filter & Central $\lambda(\mu \mathrm{m})$ & Coverage $(\mu \mathrm{m})$ \\
\hline$J$ & 1.25 & $0.97-1.07$ & $\mathrm{~Pa} \beta$ & 1.282 & $\sim 0.1$ \\
$H$ & 1.65 & $1.49-1.78$ & $\mathrm{Br} \gamma$ & 2.16 & $\sim 0.1$ \\
$K_{\mathrm{s}}$ & 2.15 & $1.99-2.30$ & $\mathrm{H}_{2}(2-1)$ & 2.24 & $\sim 0.1$
\end{tabular}

show different kinematic behaviour (Maíz Apellániz et al. 2004; Tenorio-Tagle et al. 2000; Sabalisck et al. 1995; Relaño \& Kennicut 2009).

Aiming to characterize the youngest stellar population and its environment, we performed near-infrared (NIR) photometry $(J H K)$ and analysed narrow-band images in $\mathrm{Pa} \beta, \mathrm{Br} \gamma$ and $\mathrm{H}_{2}(2-1)$. Taking into account that NIR observations are less affected by dust extinction characteristic in star-fomation environments, we can take a deep dive into NGC 604 to study those very young objects which are still immersed in their parental clouds at the sites of current star formation.

\section{Images and data processing}

The images were obtained with the Near InfraRed Imager and Spectrometer (NIRI) at Gemini North. The resulting plate scale is 0.117 arcsec pixel $^{-1}$, with a field of view of $120 \times 120 \operatorname{arcsec}^{2}$. The filters used and their main characteristics are listed in Table 1 .

The images were taken under excellent seeing conditions, on average $\sim 0.35^{\prime \prime}$ in the $J, H$ and $K_{\mathrm{s}}$ images. A set of approximately 10 individual exposures was taken in each band to combine into the final image. Data reduction and processing were performed with specific tasks using the GEMINI-NIRI IRAF package. Images were sky subtracted and flat fielded and short dark exposures were used to identify bad pixels. Stellar magnitudes were obtained by point-spread-function (PSF) fitting in crowded fields using the DAOPHOT software (Stetson 1987) in IRAF. Although a standard procedure, PSF construction and fitting involves an iterative and careful process in which several tries were made to get the best results. The effective area covered by our photometry is $\sim 107 \times 107 \operatorname{arcsec}^{2}$ $\left(\sim 430 \times 430 \mathrm{pc}^{2}\right.$ at the distance of M33). The average photometric errors are 0.09, 0.11 and 0.21 mags in the $H, J$ and $K_{\mathrm{s}}$ filters, respectively, and the completeness limits are $22 \mathrm{mag}$ in $J$ and $21 \mathrm{mag}$ in $H$ and $K_{\mathrm{s}}$. Magnitudes in the individual filters were matched in a unique list containing 5566 objects in the field in which all three $J, H$ and $K_{\mathrm{s}}$ magnitudes were measured. Astrometry was derived using 35 objects in common in our field of NGC 604 and the GSC-II Catalog, Version 2.3.2 (2006) in the ICRS, Equinox J2000.0.

\section{Results and discussion}

The resulting color-magnitude $(\mathrm{CM})$ and color-color $(\mathrm{CC})$ diagrams are shown in Figures 1 and 2, respectively. We have included $\sim 2000$ selected objects, located within a radius of $48 \operatorname{arcsec}(\sim 200 \mathrm{pc})$ and centered on NGC 604, meeting a certain photometric quality level [magnitude error $\leqslant$ median(error) $+1.0 \times$ standard deviation(error)]. Red symbols in both plots are objects that lie on the right side of the reddening line for a O6-O8 V star. For each of these objects, the error in $\left(H-K_{\mathrm{S}}\right)$ color is smaller than its distance to the reddening line, so that we can ensure that they undoubtedly show an IR excess. 

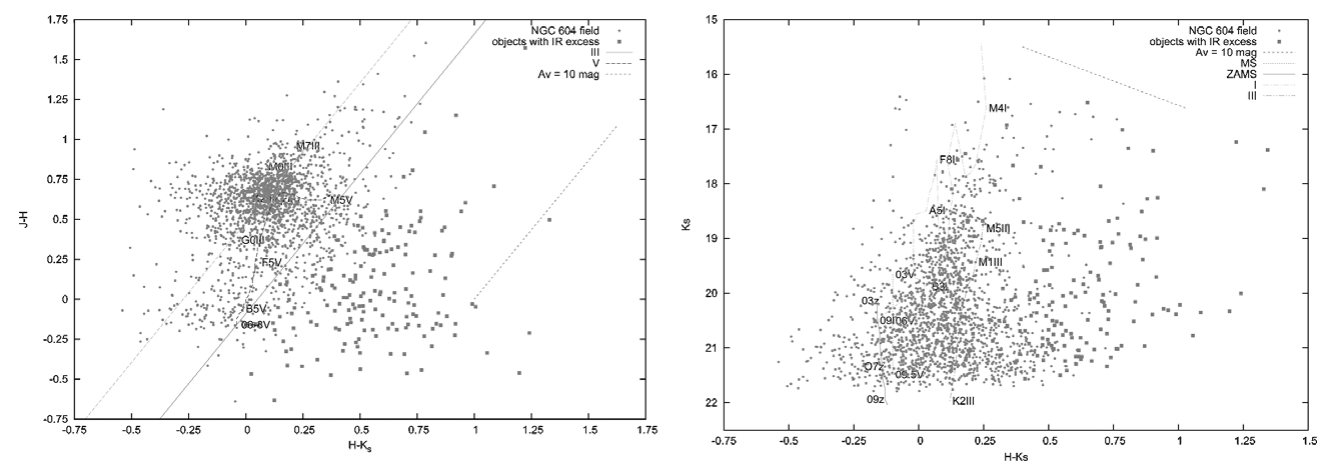

Figure 1. Color-color (left) and color-magnitude (right) diagrams for objects observed in a circular area centered on NGC 604. Red squares are objects that show an IR excess.

Among the objects with intrinsic IR excesses in GHRs we expect to find Wolf-Rayet stars, early supergiants and Of stars, and massive young stellar objects (MYSOs). Many of these types of objects (or candidates) have already been found in NGC 604. Based on our deep JHK photometry we found a total of 164 objects that show IR excesses. As can be expected, $\sim 70 \%$ of these lie in a small area near the region's center and a large proportion are tightly grouped in regions coincident with the radio-continuum peaks at $8.4 \mathrm{GHz}$ from Churchwell \& Goss (1999), as shown in Figure 3 (left panel). These results are in complete agreement with the analysis of Barbá et al. (2009) based on HST/NICMOS NIR images, where MYSO candidates also appeared aligned with the radio peak structures.

What we found supports the idea mentioned by many authors, that those areas may be embedded star-forming regions, also taking into account that regions coincident with the radio knots show conditions of high temperature and density (Tosaki et al. 2007), and in two of them Maíz Apellániz et al. (2004) identified compact HiI regions. Most objects with IR excesses will be the targets for further observing programs (making use of the integral-field spectroscopic facilities at Gemini North) to elucidate their nature and derive accurate properties by means of spectroscopic study. Also, those objects that
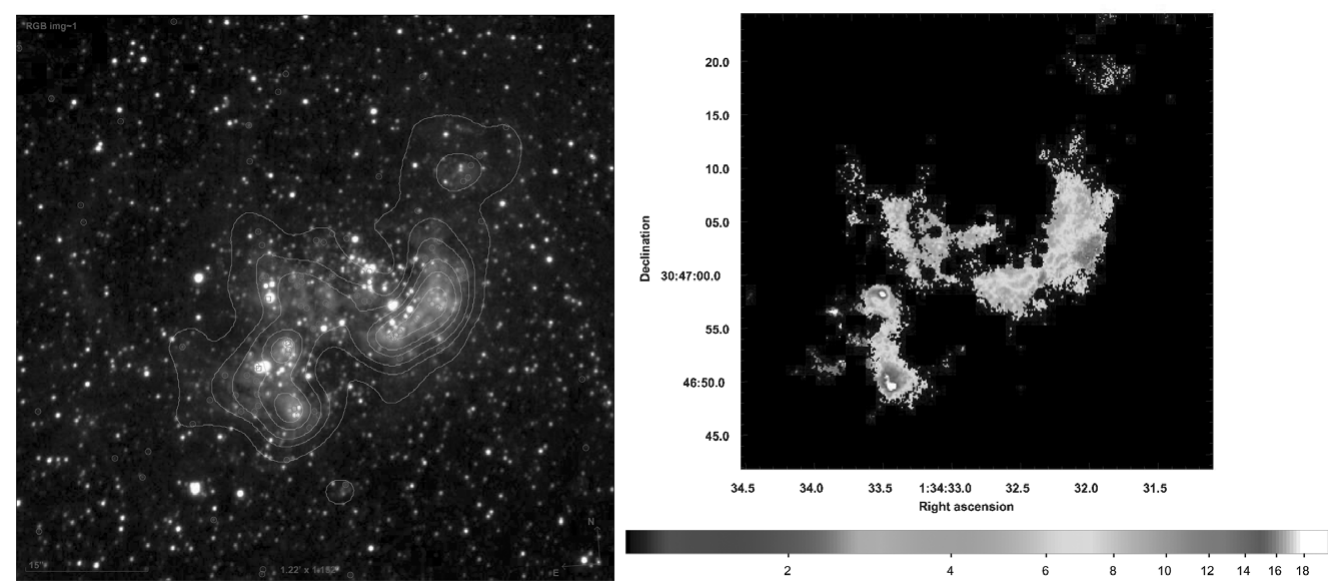

Figure 2. (left) Three-color $\left(J, H\right.$ and $K_{\mathrm{s}}$ ) composite image of NGC 604 with $8.4 \mathrm{GHz}^{\mathrm{radio}-}$ continuum countours overlaid (adapted from Churchwell \& Goss 1999). Red circles are objects that show IR excess in our photometry. (right) Extinction map from $\mathrm{Br} \gamma / \mathrm{H} \alpha$, regions with higher extinction are red/white. 
are located at the bright, massive end on the CM diagram deserve further observation and study.

On the basis of our narrow-band images we generated an extinction map, derived from the observed variations of the $\mathrm{Br} \gamma$ to $\mathrm{H} \alpha$ ratio (with the $\mathrm{H} \alpha$ image taken from Bosch et al. 2002). The color scale used in the map shows regions with higher extinction in red/white. The present and future results of our NIR study will be placed in the context of previous studies of NGC 604 to complete the picture of the overall formation and evolution scenario of this GHR.

\section{Acknowledgements}

RB acknowledges partial support from the Universidad de La Serena, project DIULS CD08102.

\section{References}

Barbá, R. H., Maíz Apellániz, J., Pérez, E., Rubio, M., Bolatto, A., Fariña, C., Bosch, G., \& Walborn, N. R. 2009, in: E. Pérez, R. de Grijs \& R. M. González-Delgado (eds.), Young massive star clusters: initial conditions and environments, ApSS, 324, 309

Bosch, G., Terlevich, E., \& Terlevich, R. 2002, MNRAS, 329, 481

Bruhweiler, F. C., Miskey, C. L., \& Smith Neubig, M. 2003, AJ, 125, 3082

Churchwell, E. \& Goss, W. M. 1999, ApJ, 514, 188

Conti, P. S. \& Massey, P. 1999, ApJ, 249, 471

Díaz, A. I., Terlevich, E., Terlevich, R., González-Delgado, R. M., Pérez, E., \& García-Vargas, M. L. 1996, in: C. Leitherer, U. Fritze-von Alvensleben \& J. Huchra (eds.), From Stars to Galaxies: The Impact of Stellar Physics on Galaxy Evolution, ASP Conf. Ser., 98, 399

D’Odorico, S. \& Rosa, M. 1981, ApJ, 248, 1015

Drissen, L., Moffat, A. F. J., \& Shara, M. M. 1993, AJ, 105, 1400

González Delgado, R. M. \& Pérez, E. 2000, MNRAS, 317, 64

Hunter, D. A., Baum, W. A., O’Neil Jr., E. J., \& Lynds, R. 1996, ApJ, 456, 174

Maí Apellániz, J., Pérez, E., \& Mas-Hesse, J. M. 2004, AJ, 128, 1196

Relaño, M. \& Kennicutt, R. C. 2009, ApJ, 699, 1125

Sabalisck, N. S. P., Tenorio-Tagle, G., Castaneda, H. O., \& Muñoz-Tuñón, C. 1995, ApJ, 444, 200

Stetson, P. B. 1987, PASP, 99, 191

Tenorio-Tagle, G., Muñoz-Tuñón, C., Pérez, E., Maíz Apellániz, J., \& Medina-Tanco, G. 2000, ApJ, 541, 720

Terlevich, E., Díaz, A. I., Terlevich, R., González-Delgado, R. M., Pŕez, E., \& García-Vargas, M. L. 1996, MNRAS, 279, 1219

Tosaki, T., Miura, R., Sawada, T., Kuno, N., Nakanishi, K., Kohno, K., Okumura, S. K., \& Kawabe, R. 2007, ApJ, 664, 27 